\title{
Verzeichnis der Gesellschaften
}

Aachener und Münchener Lebensversicherungs-Aktiengesellschaft, Karlsruhe i. B., Ritterstraße 3, Ruf 50 40/42, Drahtwort: Lebensschutz. Hauptverwaltung Berlin: Berlin-Schöneberg, Badensche Straße 2, Ruf 714753 . . . . . . . . . .

Agrippina Lebensversicherungs-Aktiengesellschaft, Berlin W 30 , Mackensenstr. 16, Ruf 2416 65/67, Drahtwort: Agrippinaleben, Zweigniederlassung: Köln, Riehler 90, Str. Ruf 73723 u. 74786

Albingia Lebensversicherungs-Aktiengesellschaft, Hamburg 1, Ballindamm 39, Ruf 3210 02, Drahtwort: Albingia, Fernschreiber 0211774 . . . . . . . . . . . . . . . . . . . . .

Allgemeine Assekuranz (Assicurazioni Generali), Triest. Hauptbevollmächtigter für Deutschland: Carl Rieck, Hamburg 1, Monckebergstraße 31, Ruf $330355 / 56$, Drahtwort: Versrieck

Allgemeine Lebens- und Aussteuer-Versicherungs-Aktiengesellschaft (vormals Gisela, Deutsche Lebens- und Aussteuer-Versicherungs-Aktiengesellschaft), München, siehe unter Gisela Allgemeine Lebens- und Aussteuer-Versicherungs-Aktiengesellschaft ...................

Allgemeine Rentenanstalt Lebens- und Rentenversicherungs-AG., Stuttgart-S, Tübinger Straße 22, Ruf $77351 / 53$, Drahtwort: Rentenanstalt. Bezirksdirektion Berlin : Berlin SW 29, Gneisenau-

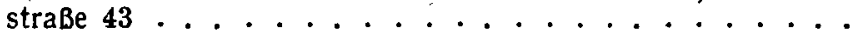

Allianz Lebensversicherungs-AG., Hauptverwaltung: Stuttgart $W$, Silberburgstr. 174, Ruf $68141 / 68147$, Fernschreiber 0723571 . Berlin-Charlottenburg 2, Jebensstraße 1, Ruf 3252 51, Fernschreiber 028 810. München 22, Ludwigstraße 12, Ruf 28431 , Fernschreiber 063 791. Drahtwort: Allianzleben . . . . . . .

Alte Leipziger Lebensversicherungsgesellschaft auf Gegenseitigkeit, Frankfurt a. M., Bockenheimer Landstraße 42, Ruf 71451 , Drahtwort: Alteleip. Bezirksdirektion GroB-Berlin: BerlinSchöneberg, Martin-Luther-Straße 54, Ruf 710148. . . . .

Alte Volksfürsorge Gewerkschaftlich-Genossenschaftliche Lebensversicherungsaktiengesellschaft, Hamburg 1, An der Alster 57/61, Ruf 248051 , Drahtwort: alvovers, Fernschreiber 021 1300. Geschäftsstelle Berlin: Berlin-Wilmersdorf, Hohenzollerndamm 174/177, Ruf 874782 . . . . . . . . . . . . . . . .

Atlas Lebensversicherungs-Aktiengesellschaft, Heidelberg, Treitschkestraße 3, Ruf 3293, Drahtwort: Atlas ... . . .

Basler Lebens-Versicherungs-Gesellschaft, Basel, Albananlage 7, Ruf 221830 . Hauptverwaltung für die Bundesrepublik Deutschland: Frankfurt a.M., MendelssohnstraBe 80, Ruf 738 40, Drahtwort: Baslerleben. Zweigniederlassung: Berlin W 15, Kurfürstendamm 52 (Haus Basel), Ruf 912844 . . . . . . . . . . .

Bayerische Beamtenversicherungsanstalt, Allgemeiner Lebensversicherungsverein auf Gegenseitigkeit, München 2, Lenbachplatz 4, Ruf 524 41-45, Drahtwort: Beamtenleben. Bezirksdirektion Berlin: Berlin-Charlottenburg 2, Berliner Straße 153, Ruf 346052 
Berlinische Lebensversicherungs-Gesellschaft Aktiengesellschaft, Berlin SW 68, Markgrafenstraße 11, Ruf 6104 71, Wiesbaden, Schillerplatz 2, Ruf 598 16, Drahtwort: Berlinische .

Bonner Lebensversicherung Aktiengesellschaft, Köln, Hansaring 42-46, Ruf 225642

Braunschweigische Lebensversicherung A. G., Braunschweig, Jasperallee 86/87, Ruf 254 51/52/53, Drahtwort: 25451 Braunleben. Bezirksdirektion Berlin: Berlin-Schöneberg, Innsbrucker Straße 28, Ruf 711365 .

Concordia Lebensversicherungs-Aktiengesellschaft, Köln, MariaAblaB-Platz 1, Ruf 29 01, Drahtwort: Concordialeben, Fernschreiber 088666 , Verwaltung Großberlin: Berlin W 15, Kurfürstendamm 52, Ruf $914553 / 54$.

Debeka Sterbegeld- und Lebensversicherungsverein auf Gegenseitigkeit, Koblenz, Süd-Allee 15-19, Ruf 2375, Drahtwort: Debeka, Geschäftsstelle Berlin: Berlin-Wilmersdorf, Berliner Straße 36, Ruf $872472 / 73$.

Der Anker Allgemeine Versicherungs-Aktiengesellschaft, Wien I, Hoher Markt 12, Direktion für Deutschland: Frankfurt a. M., Friedrich-Ebert-Straße 72, Ruf 32140, Filialdirektion Berlin: Berlin W 15, Meinekestraße 5, Ruf 912779

Deutsche Anwalt- und Notar-Versicherung Lebensversicherungsverein auf Gegenseitigkeit, siehe unter Hansa Lebensversicherung a. G. .

Deutsche Ärzteversicherung auf Gegenseitigkeit, Berlin-Zehlendorf, Potsdamer Straße 47/48, Ruf 8435 36/37, Hamburg 1, An der Alster 49, Ruf 244047

Deutsche Beamten-Versicherung Offentlichrechtliche Lebensund Renten-Versicherungsanstalt, Berlin W 15, Knesebeckstraße 59/60, Ruf $914144 / 45 / 46$, Drahtwort: Beamtenleben, Westzonenhauptverwaltung: München 22, Von-der-Tann-Str. 2, Ruf 23745

Deutsche Eisenbahn-Versicherungskasse Lebensversicherungsverein a. G., Sitz Berlin, Hauptleitung Köln, An der Münze 12-18, Dienstfernruf $943 / 2285$, Postfernruf 72859 , Bezirksleitung Berlin: Berlin W 35, Kluckstraße 25, Ruf 244788 .

Deutsche Reichsbahn-Sterbekasse Lebensversicherungsverein a. G. siehe Deutsche Eisenbahn-Versicherungskasse, Lebensversicherungsverein a. G.

Deutscher Herold Volks- und Lebensversicherungs-AG., Bonn, Poppelsdorfer Allee 31/33, Ruf 38451 , Drahtwort: Heroldleben, Berlin SW 48, Friedrichstraße 219/220, Ruf 248751 ... .

Deutscher Lloyd Lebensversicherung Aktiengesellschaft, München 15, Bayerstraße 47, Ruf 57021 , Drahtwort: Lloydleben. Hauptverwaltung Berlin: Berlin-Friedenau, Bornstraße 1, Ecke Bundesallee (Lloydhaus), Ruf $839561 / 62$. . . . . .

Deutscher Ring Lebensversicherungs-Aktiengesellschaft, Hamburg 36, Karl-Muck-Platz 1, Ruf 3414 41, Drahtwort: Ringsicherung. Filialdirektion GroB-Berlin: Berlin W 30, Kurfürstenstraße 133, Ruf $242551 / 52$.. . . . . . . . . . .

Eos Volks- und Lebensversicherungs-Aktiengesellschaft, Düsseldorf, Hauptverwaltung Coburg (Bayern), Herrngasse 11, Ruf 35 49, Drahtwort: Eos. Bezirksdirektion Berlin: Berlin W 30, Geisbergstraße 11, Ruf 245253 
Friedrich Wilhelm Lebensversicherungs-Aktiengesellschaft, BerlinWilmerdorf, Hohenzollerndamm 196, Ruf 870346 . Köln, Enggasse, Ruf 2861, Drahtwort: Friedhelmleben, Fernschreiber 088587

Gerling-Konzern Lebensversicherungs-Aktiengesellschaft, Köln, Gerling-Hochhaus, Ruf 2861, Drahtwort: Gerlingleben, Fernschreiber 088 587, Zweigniederlassung Berlin: Berlin-Wilmersdorf, Hohenzollerndamm 196, Ruf 870346 . . . . . . . .

Gilde Deutsche Versicherungs-Aktiengesellschaft, Düsseldorf, Graf-Recke-Straße 82, Ruf 601 85, 631 52, 64924 , Geschäftsstelle Berlin: Berlin W 15, Düsseldorfer Straße 33, Ruf 913771

Gisela Allgemeine Lebens- und Aussteuer-Versicherungs-Aktiengesellschaft, München 8, Prinzregentenplatz 7/9, Ruf 449061 , Drahtwort: Giselaversicherung, Geschäftsstelle Berlin: BerlinWilmersdorf, Rudolstädter Straße 121, Ruf 874767 . . . .

Gladbacher Lebensversicherung Aktien-Gesellschaft, MönchenGladbach, Wallstraße 30/32, Ruf 2 45 51, Drahtwort: Gladbachleben, Geschäftsstelle Berlin: Berlin-Lichterfelde-W, Enzianstraße 2, Ruf 760626 .

Gothaer Lebensversicherung auf Gegenseitigkeit, Göttingen, Gothaer Platz 8, Ruf 38 71, Drahtwort: Gothaleben, Hauptbevollmächtigter für Berlin: Berlin W 15, Kurfürstendamm 54/55, Ruf 911265

Hamburg-Mannheimer Versicherungs-Aktien-Gesellschaft, Hamburg 36, Alsterufer 1, Ruf 4411 01, Drahtwort: Hamavag, Filialdirektion Berlin: Berlin W 15, Meinekestr. 3, Ruf 912850

Hannoversche Lebensversicherung auf Gegenseitigkeit zu Hannover, vormals Preußischer Beamten-Verein, Raschplatz 13, Ruf 242 46, Drahtwort: Hannoverleben, Büro Berlin W 15, Kurfürstendamm 224, Ruf 914859 . . . . . . . . . .

Hansa Lebensversicherung a. G., Hamburg 36, Alsterufer 1, Ruf 441101 , Drahtwort: Hansaleben, Filialdirektion Berlin: Berlin W 15, Meinekestraße 3, Ruf 912850 . . . . . . . .

Iduna-Germania Lebens-Versicherungsgesellschaft auf Gegenseitigkeit in Berlin, Berlin SW 68, Charlottenstraße 13, Ruf 610236 , Drahtwort: Idunaleben, Fernschreiber $028806-$ Hamburg 36, Alsterufer 50, Ruf 4411 41, Fernschreiber 021397

Isar Lebensversicherungs-Aktiengesellschaft, München 23, Giselastraße 21/23, Ruf 39221, Drahtwort: Isarag, Filialdirektion Berlin: Berlin W 30, Geisbergstraße 11, Ruf 244272 . . .

Karisruher Lebensversicherung A. G., Karlsruhe i. B., Helmholtzstraße 1, Ruf $5300 / 04$, Bezirksdirektion GroB-Berlin: Berlin W 30, HardenbergstraBe 15, Ruf 322282

Kölnische Lebensversicherung auf Gegenseitigkeit, Köln, Clever StraBe 36, Ruf 749 48, Bezirksdirektion Berlin: Berlin-Lichtenrade, Lintruper Straße 13, Ruf 708479 . . . . . . . . . .

Lebensversicherungsanstalt Berlin, Körperschaft des öffentlichen Rechts, Berlin W 35, Am Karlsbad 4/5, Ruf 240012 . . . .

Leipziger Verein-Barmenia Lebensversicherung auf Gegenseitigkeit, Hamburg-Volksdorf 1, Halenreie 40-44, Ruf 2044 41-43, Drahtwort: Elvaubeleben, Bezirksdirektion Berlin: Berlin-Charlottenburg 4, Kantstraße 46, Ruf 326077 . . . . . . . . .

Magdeburger Allgemeine Lebens- und Rentenversicherungs-Aktiengesellschaft, Darmstadt, Rheinstraße 14, Ruf 30 23, Drahtwort: Allgema. Vom 1. Januar 1954 an: Fulda, Heinrich-von-BibraPlatz, Niederlassung Berlin: Berlin W 30, Bamberger Straße 44, Ruf 244019 . . . . . . . . . . . . . . . . . . . . . . 
Magdeburger Lebens-Versicherungs-Gesellschaft, Köln, Enggasse, Ruf 28 61, Drahtwort: Altemagdeburger, Fernschreiber 088 587, Büro Berlin: Berlin-Wilmersdorf, Hohenzollerndamm 196, Ruf 870346

Mannheimer Lebensversicherungs-Gesellschaft A.-G., Berlin W 30, Rankestraße 23, Ruf 2424 14/15, Mannheim E 5, Technisches Rathaus, Ruf $31613 / 15$. . . . . . . . . . . . .

Münchener Lebensversicherungsanstalt AG, München 23, Leopoldstraße 6 , Ruf 35091,32158 , Drahtwort: Müleba . . . .

Münchener Verein Lebens-' und Altersversicherungsanstalt auf Gegenseitigkeit, München 2, Pettenkoferstraße 19, Ruf 50441

National Lebensversicherungs-Aktien-Gesellschaft, Lübeck, Travemünder Allee 9, Ruf 25321 , Drahtwort: National, Fernschreiber 026 850, Direktion für Groß-Berlin: Berlin SW 68, Friedrichstraße 31, Ruf 617627

Neue Welt Lebensversicherungs-Aktiengesellschaft, Hamburg, siehe unter Deutscher Ring, Lebensversicherungs-Aktiengesellschaft . . . . . . . . . . . . . . . .

Nord-Deutsche Lebensversicherungs-Aktiengesellschaft, Hamburg 11, Alterwall 12, Ruf $341301 / 06$, Drahtwort: Nordleben, Hauptverwaltungsstelle Berlin: Berlin-Charlottenburg, Württembergallee 1, Ruf 976526 und 978732 ........

Nordstern Lebensversicherungs-Aktiengesellschaft, Berlin-Schöneberg, Nordsternplatz, Ruf 7103 31, Drahtwort: Nordsternleben, Fernschreiber 028511 , Köln, Gereonstraße 43-55, Ruf 735 45/47, Fernschreiber 088731 . . . . . . . . . . .

Nürnberger Lebensversicherung AG, Nürnberg, Rathenauplatz 16-18, Ruf 514 41, Drahtwort: Lebensbank, Fernschreiber 06/2163. Geschäftsstelle Berlin: Berlin-Charlottenburg 1, Berliner StraBe 137, Ruf 324979 . . . . . . . . . . . . .

Oeffentlich-rechtliche Lebensversicherungsanstalten . . . . 65 Verzeichnis der einzelnen Anstalten siehe Seite 65b

Pensionsverein Deutscher Pensionszuschuß und Lebensversicherungsverein a. G., München 15, Beethovenplatz 4, Ruf

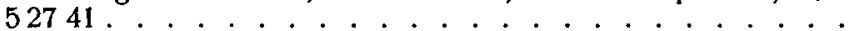

Prima Lebensversicherungsverein a. G., Nürnberg 2, Frauentorgraben 11/13, Ruf 2 75 54, Drahtwort: Prima . . . . . . .

'Raiffeisendienst Lebensversicherungsgesellschaft a. G., BerlinCharlottenburg 2, Schillerstraße 3, Ruf 323811 . Wiesbaden, Sonnenberger Straße 2a, Ruf 59331

Rheinisch-Westfălische Lebensversicherung a. G., WuppertalBarmen, Carnaper StraBe 73-75, Ruf $56667 / 8$, Direktionsgeschäftsstelle Berlin: Berlin-Friedenau, Ringstraße 61/62,

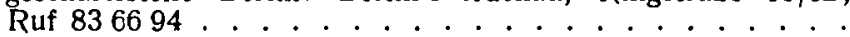

Rothenburger Lebensversicherungs-AG, Köln, Maria-AblaBPlatz 15, Ruf 2123 41, Drahtwort: Rothleben, Bezlrksdirektion Berlin: Berlin W 15, Kurfürstendamm 52, Ruf 912885 . . . .

Schweizerische Lebensversicherungs- und Rentenanstalt, Zürich, Alpenquai 40. Niederlassung für Deutschland: München 23, Leopoldstraße 8, Ruf $34251 / 53$, Drahtwort: Schweizerische München . . . . . . . . . . . . . . . . . . . . . . . .

Sparkassen-Versicherung AG., Stuttgart-N, Friedrichstraße 4, Ruf 95646 . . . . . . . . . . . . . .

Terra Lebensversicherung Aktiengesellschaft, Berlin-Schöneberg, siehe unter Albingia, Lebensversicherungs-Aktiengesellschaft 
Verband offentlicher Lebensversicherungsanstalten in Deutschland, Duisseldorf, Friedrichstraße 62-74, Ruf 84 237, Drahtwort: Lebenverband, Verwaltungsstelle Berlin: Berlin SW 11, Stresemannstraße 62-64, Ruf 667488 . . . . . . . . . .

Vereinigte Lebensversicherungsanstalt a. G. für Handwerk, Handel und Gewerbe, Hamburg 36, Alsterufer 50, Ruf 4411 41, Drahtwort: Vereinigteleben, Fernschreiber 021 1397, Bezirksdirektion Berlin: Berlin-Steglitz, Schloßstr. 30, Ruf 721637. . . . .

Victoria zu Berlin Allgemeine Versicherungs-Actien-Gesellschaft, Berlin SW 68, Lindenstraße 20-25, Ruf 6101 01, Drahtwort: Victoriabank, Fernschreiber 028477 , Zonenhauptverwaltung für den Westen: Düsseldorf, Bahnstraße 2-8, Ecke Königsallee, Ruf 87 11, Fernschreiber 082.984 . . . . . . . . . .

Victorla am Rhein Allgemeine Versicherungs-Actien-Gesellschaft, Düsseldorf, Bahnstraße 2-8, Ecke Königsallee, Ruf 87 11, Drahtwort: Victoriarhein, Fernschreiber 082984 . . . . . . . .

Vita Lebensversicherungs-Aktiengesellschaft, Zürich, Mythenquai 10, Ruf (051) 2748 10. Zweigniederlassung für Deutschland: Frankfurt a.M., Friedrich-Ebert-Straße 3-5, Ruf 906 01, Zweigniederlassung Berlin: Berlin-Charlottenburg, Leibnizstraße 63, Ruf 320697 .................

Vohk Lebensversicherungsanstait ostdeutscher Handwerkskammern, V. a. G., zu Berlin, Berlin W 35, Potsdamer StraBe 76, Ruf $246734 / 35$, Zweigniederlassung: Kiel, Holstenbrücke 4-6, Ruf 5395

Volkshilfe Lebensversicherungs-Aktiengesellschaft, Berlin-Friedenau, SarrazinstraBe 11-15, Ruf 832055 , Fernschreiber 028588 , Köln, Kaiser-Wilhelm-Ring 46, Ruf $211641 / 43$, Fernschreiber 088862

Volkswohl-Bund Lebensversicherung a. G., Berlin W 35, Schoneberger Ufer 59, Ruf 249161 , Hauptverwaltung in den Westzonen: Dortmund, Sudwall 39, Ruf $22441 / 43$. . . . . . .

Winterthur Lebensversicherungs-Gesellschaft in Winterthur (Schweiz), Direktion für die Bundesrepublik: München 23, Leopoldstraße 34-36, Ruf 360721 , Fernschreiber 063378 .

Witwen- und Waisenkasse des Reichs- und Staatsdienstpersonals Allgemeine Lebensversicherungsanstalt a. G., München 2, Marsstraße 22-23a, Ruf 58491 .............

Württembergischer Versicherungsverein a. G., Stuttgart-S, MörikestraBe 5, Ruf $76236 / 38$, Drahtwort: Württleben . . 64

Verband der Lebensversicherungsunternehmen e. V.

Bonn, Bonner Talweg 33, Ruf $33751 / 52$,

Drahtwort: Lebensverband.

Verbandsdirektor: Dr. Waldemar Adler. 
